\title{
Comments on the black hole information problem
}

\author{
David A. Lowe* \\ Department of Physics, Brown University, Providence, RI 02912, USA \\ Lárus Thorlaciust \\ Science Institute, University of Iceland, \\ Dunhaga 3, IS-107 Reykjavik, Iceland
}

\begin{abstract}
String theory provides numerous examples of duality between gravitational theories and unitary gauge theories. To resolve the black hole information paradox in this setting, it is necessary to better understand how unitarity is implemented on the gravity side. We argue that unitarity is restored by nonlocal effects whose initial magnitude is suppressed by the exponential of the Bekenstein-Hawking entropy. Time-slicings for which effective field theory is valid are obtained by demanding the mutual back-reaction of quanta be small. The resulting bounds imply that nonlocal effects do not lead to observable violations of causality or conflict with the equivalence principle for infalling observers, yet implement information retrieval for observers who stay outside the black hole.
\end{abstract}

*Electronic address: lowe@brown.edu

${ }^{\dagger}$ Electronic address: lth@hi.is 


\section{INTRODUCTION}

The black hole information paradox [1] highlights an incompatibility between locality in spacetime physics and unitarity in quantum theories [2, 3]. The argument for information loss is made in the context of a low-energy effective theory on a semiclassical background spacetime, which is assumed to be a conventional local quantum field theory. Unitarity in black hole evolution, on the other hand, implies nonlocality in spacetime and the nonlocal effects in question must act over macroscopic length scales.

There is mounting evidence that the paradox is resolved in favor of unitarity, coming for example from matrix theory [4], the AdS/CFT correspondence [5, 6, 7], and more indirectly from the microscopic computation of black hole entropy [8]. The AdS/CFT correspondence in particular provides a framework where unitarity is manifest on the gauge theory side, but since a semiclassical geometry is only recovered in a strong coupling limit, the implementation of unitarity on the gravity side remains a challenge. One must somehow reconcile the semiclassical effective field theory with the apparent duplication of degrees of freedom required for the information carried by matter falling into the black hole to be returned in the Hawking radiation.

These concerns only arise if one assumes that the evaporation process is governed by a local effective field theory combining matter and gravity. In particular, it is assumed that one can construct so called nice time slices [9, 10] that simultaneously intersect most of the outgoing Hawking radiation and an infalling observer having just passed inside the horizon in such a way that both the outgoing radiation and the infalling observer have low energy in the local frame of the slice, as indicated in figure 1. These slices can be constructed to avoid regions of large spacetime curvature, except near the black hole endpoint.

It was argued in 10, 11], based on perturbative string theory, that the effective field theory on such time slices is in fact nonlocal on macroscopic length scales. In a similar vein, 12, 13] argued that gravitational back-reaction will undermine locality in the effective field theory on nice time slices in a black hole geometry. We can turn these arguments around to say that effective field theory is only to be applied on restricted time slices. In the limit that we treat the near-horizon region of a large black hole as Rindler space, we formulate the restriction in terms of an upper bound on the relative boost of any two observers, that are at rest with respect to different parts of a time slice. When the restriction due to the 


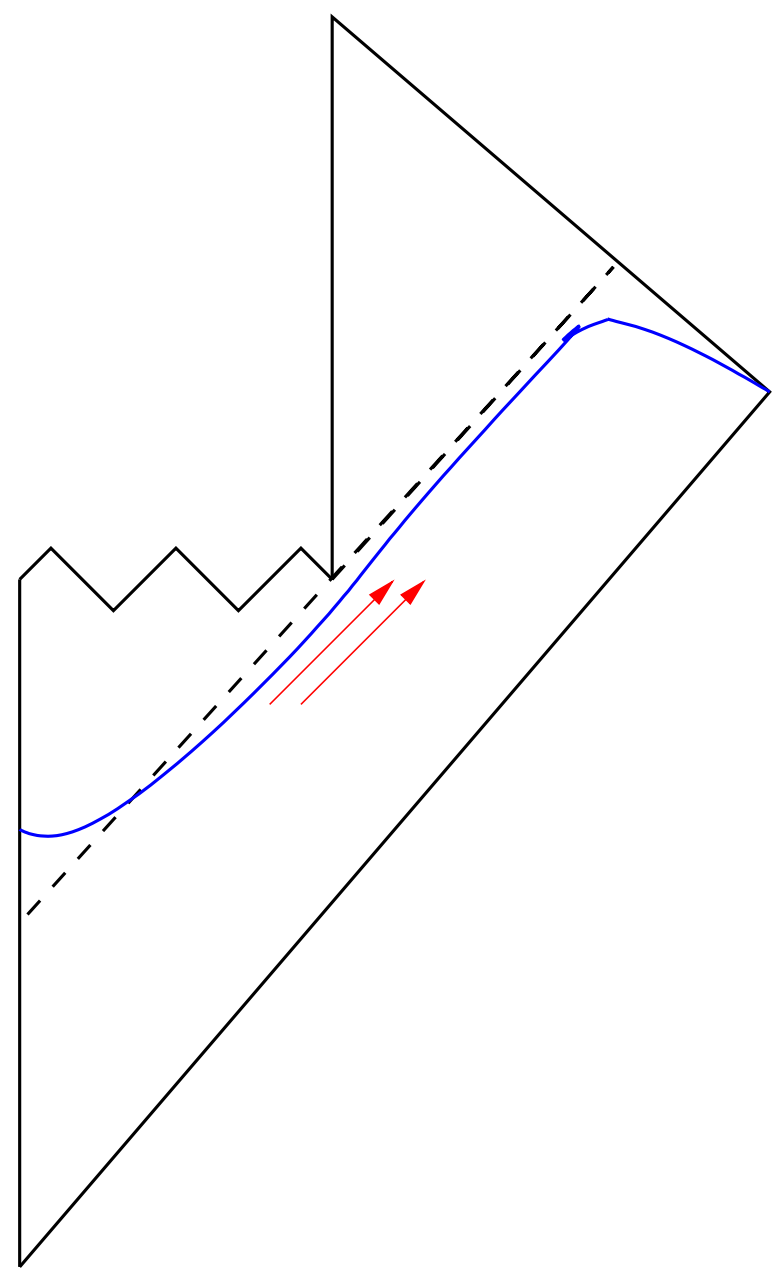

Figure 1: Timeslices can be constructed in the semiclassical geometry that simultaneously intersect most of the Hawking radiation and a freely falling observer who crosses the horizon early on.

boost bound is taken into account, we find that observers crossing the horizon of a black hole will see local and causal physics to within the finite accuracy of their measuring apparatus until they approach the singularity, while outside observers can see complete retrieval of information.

\section{THE PARADOX}

We begin with a sharp statement of the black hole information paradox. Consider a pair of spin $1 / 2$ particles in a singlet state

$$
\frac{1}{\sqrt{2}}\left(\left|\uparrow_{1}\right\rangle\left|\downarrow_{2}\right\rangle-\left|\downarrow_{1}\right\rangle\left|\uparrow_{2}\right\rangle\right)
$$


where $|\uparrow\rangle,|\downarrow\rangle$ refer to eigenvectors with spin oriented along the $z$-axis. Spin \#2 is kept far outside the black hole in the custody of observer $\mathcal{O}_{\text {far }}$, while spin \#1 is sent, along with a free-falling observer $\mathcal{O}_{\text {free }}$, into the black hole. Another observer $\mathcal{O}_{\text {accel }}$, hovers outside the black hole and measures the Hawking radiation coming off. Far away from the black hole $\mathcal{O}_{\text {far }}$ conducts a measurement on spin \#2, which consists of either measuring the spin along the $z$-axis or the $x$-axis. If the other observers can combine their results to determine which of these possibilities has taken place, then information has been acausally transmitted over an arbitrarily long distance.

Suppose the Hawking radiation eventually carries all the information about the internal state of the black hole. Then $\mathcal{O}_{\text {accel }}$ can conduct a measurement of the radiation to effectively determine the component of spin \#1 along the $z$-axis. If $\mathcal{O}_{\text {far }}$ measures spin \#2 up along the $z$-axis, the indirect measurement carried out by $\mathcal{O}_{\text {accel }}$ will give spin down. If $\mathcal{O}_{\text {far }}$ instead measures spin \#2 along the $x$-axis there will be no correlation between the results of the two spin measurements. So far no actual information has been acausally propagated, since the probability for each observer to measure spin up or down along any axis is $50 \%$. Therefore $\mathcal{O}_{\text {accel }}$ cannot know in which direction $\mathcal{O}_{\text {far }}$ conducted the measurement. Only if they were to later come into causal contact and compare notes, would they notice the nonlocal EPR correlation between their spin measurements.

Now suppose $\mathcal{O}_{\text {free }}$ makes a measurement on spin $\# 1$ along the $z$-axis. Since $\mathcal{O}_{\text {free }}$ is at spacelike separations from $\mathcal{O}_{\text {accel }}$ their measurements should commute with each other if local quantum field theory is valid. Therefore $\mathcal{O}_{\text {free }}$ will measure spin up or down with $50 \%$ probability. Again no information has acausally propagated, despite the nonlocal EPR correlations between spins \#1 and \#2.

Finally, $\mathcal{O}_{\text {accel }}$ enters the black hole, and compares notes with $\mathcal{O}_{\text {free }}$. If they disagree on the outcome of their experiments, they know with certainty that $\mathcal{O}_{\text {far }}$ must have conducted a measurement along the $x$-axis. Thus with some finite probability, acausal communication has occurred between $\mathcal{O}_{f a r}$ and our intrepid observers inside the black hole. In essence, the black hole operates as a quantum information cloning machine, and such machines allow EPR correlations to be turned into EPR phones. This experiment provides us with a clear statement of the information paradox.

Due to the extreme redshifts involved, it turns out to be very difficult for the observer $\mathcal{O}_{\text {accel }}$ to receive any signal from observer $\mathcal{O}_{\text {free }}$ before crashing into the singularity. In [14] 
it was argued that $\mathcal{O}_{\text {free }}$ would have to employ an enormous frequency for the transmission and the amount of energy involved would then drastically alter the background geometry. The argument is made in two steps. First one needs an estimate of the time $\mathcal{O}_{\text {accel }}$ has to wait outside the black hole while the indirect measurement of spin \#1 is carried out. In the second step one bounds the available time for observer $\mathcal{O}_{\text {free }}$ to perform the direct measurement on spin $\# 1$ and transmit the result for $\mathcal{O}_{\text {accel }}$ to receive before hitting the singularity.

The 3+1-dimensional estimates of [14] are easily generalized to arbitrary dimensions. The relevant facts about a $D$-dimensional black hole geometry are summarized in appendix A. If we assume the Hawking radiation and black hole are in a random pure state, as should be true if interactions effectively thermalize the black hole degrees of freedom, then we may apply the results of Page [15, 16] (see appendix B for a brief review) to compute the typical amount of information contained in the Hawking radiation. The information outflow is extremely small, of order $e^{-S_{b h}}$ where $S_{b h}$ is the Bekenstein-Hawking entropy, until one half-life of the black hole has passed, at a Schwarzschild time $t \sim r_{s} S_{b h}$ where $r_{s}$ is the Schwarzschild radius, after which the information contained in the Hawking radiation increases linearly with the thermal entropy of the radiation. The fact that $\mathcal{O}_{\text {accel }}$ has to wait such a long time before making the plunge into the black hole leaves $\mathcal{O}_{\text {free }}$ with very little time (of order $e^{-a S_{b h}}$, with $a$ an order one constant, according to the estimate at the end of appendix to perform the direct spin measurement and send off the result. Accordingly the signal would have to be encoded in a wave with frequency of order $e^{a S}$, which in turn requires more energy than is available to observers in this geometry.

\section{UNITARY QUANTUM MECHANICAL FRAMEWORK}

Let us now rerun the gedanken experiment, in the type of quantum gravity framework that AdS/CFT provides. Our key assumptions are:

1. black hole evaporation is unitary, with all the information being carried off by the Hawking radiation;

2. a quantum mechanical description of the evaporation is valid. 
We have in mind black holes whose Schwarzschild radius is large compared to any microscopic length scale but small compared to the characteristic AdS length scale. In this case, asymptotically AdS boundary conditions do not play a crucial role in our discussion, and for simplicity we will draw Penrose diagrams with asymptotically flat boundary conditions.

Suppose $\mathcal{O}_{\text {free }}$ conducts a measurement of spin $\# 1$, with result spin up. Up to the subtleties described below, having to do with the finite size of the black hole, this will project the state into

$$
\left|\uparrow_{1}\right\rangle\left|\downarrow_{2}\right\rangle .
$$

Further measurement of this state by $\mathcal{O}_{\text {accel }}$ will necessarily lead to the same answer. Likewise $\mathcal{O}_{\text {accel }}$ and $\mathcal{O}_{\text {free }}$ will agree on the results of their measurements regardless of the order with which they are conducted in some given time slicing. This can only happen if the measurement operator from the effective field theory viewpoint is nonlocal, or equivalently the degrees of freedom in the effective field theory are nonlocal. If $\mathcal{O}_{\text {accel }}$ and $\mathcal{O}_{\text {free }}$ are always in agreement, then no information is propagated acausally over arbitrary distance from $\mathcal{O}_{\text {far }}$. Likewise, no acausal propagation of information is observed by those who stay outside the black hole. Another concern is that observers inside and outside the black hole, such as $\mathcal{O}_{\text {free }}$ and $\mathcal{O}_{\text {accel }}$, are able to communicate. We reviewed the argument of [14] that this cannot happen in the previous section and the following is another variant.

We find it useful to couch the discussion in the many worlds interpretation of quantum mechanics, where one gives up the projection postulate of the Copenhagen interpretation 17, 18]. From this viewpoint the wavefunction always evolves deterministically according to the linear Schrodinger equation. This is important for the case at hand, because the outside observer conducts measurements on the entire black hole state, which includes $\mathcal{O}_{\text {free }}$. It is therefore appropriate to think of $\mathcal{O}_{\text {free }}$ as a mesoscopic quantum subsystem, whose observations do not project the quantum state. Rather, different mesoscopic states of $\mathcal{O}_{\text {free }}$ will be correlated with different values of the spin state. To the extent that the dynamics experienced by $\mathcal{O}_{\text {free }}$ is local, these different mesoscopic states will effectively decohere into quasi-classical position eigenstates due to interactions with their environment [17], so may be interpreted as measurements by $\mathcal{O}_{\text {free }}$.

Furthermore the intrinsic accuracy of measurements carried out by observers inside a black hole is limited, since the maximum size apparatus they can have access to is bounded by a subsystem with Hilbert space dimension $e^{S_{b h}}$. For example, suppose we have $N$ identical 
spins, with $2^{N}=e^{S_{b h}}$. Then the intrinsic accuracy of a spin measurement is proportional to

$$
\sigma_{\text {spin }} \sim \frac{1}{\sqrt{N}} \sim \frac{1}{\sqrt{S_{b h}}} .
$$

More generally, if $\mathcal{O}_{\text {free }}$ only has a finite time to conduct a measurement, we can assume the maximum size of the apparatus satisfies a holographic bound for the relevant causal diamond

where $A$ is the area of the relevant light-sheets $19 \mid$.

$$
\sigma_{\text {spin }} \sim \frac{1}{\sqrt{A}}
$$

As before, we invoke the results of Page [15, 16] to conclude that observer $\mathcal{O}_{\text {accel }}$ must wait for $t \sim r_{s} S_{b h}$ before useful information can be extracted from the Hawking radiation. This means for $\mathcal{O}_{\text {free }}$ to communicate results to $\mathcal{O}_{\text {accel }}$ the signal must be sent in a small trans-Planckian time

$$
t \sim e^{-a S_{b h}}
$$

with $a$ is a number of order one, else $\mathcal{O}_{\text {accel }}$ hits the singularity before receiving the signal. This implies the intrinsic accuracy in $\mathcal{O}_{\text {free }}$ measurements (44) is necessarily only of order one. Hence no useful information can be transmitted to $\mathcal{O}_{\text {accel }}$.

We can nevertheless ask whether observations on the Hawking radiation can affect the inside observer $\mathcal{O}_{\text {free }}$. Again it is helpful to keep in mind the many worlds interpretation of quantum mechanics. As the Hawking radiation moves away from the black hole, it will be an increasingly good approximation to treat its interactions as local. In the AdS/CFT correspondence the mapping to field theory degrees of freedom is best understood as the boundary is approached where it becomes local. Conversely, we expect the inside observer whose state is encoded in this Hawking radiation will experience nonlocal interactions. An outside observer can in principle employ arbitrarily large measuring apparatus, so for all practical purposes can project the black hole state into a position eigenstate of the Hawking radiation. Since the inside observer's state is itself projected, it appears that observation of the Hawking radiation in fact burns up the inside observer!

It is possible to construct so called nice time slices which intersect $\mathcal{O}_{\text {free }}$ shortly after crossing the horizon, and also intersect the bulk of the Hawking radiation near the endpoint of the evaporation. Such a time slice is indicated in figure 1 and an explicit construction is given in [10]. If we apply the decoherence story on such a time slice, the local coupling of the 
Hawking radiation to environmental degrees of freedom far from the black hole is already sufficient to burn up the freely falling observer as soon as they cross the horizon, without any explicit observation needed. While this scenario gives a consistent description for observers far from the black hole, the conclusion is distinctly undemocratic. By the equivalence principle an observer entering a large black hole in free fall is expected to experience physics as usual until the region of strong curvature near the singularity.

\section{VALIDITY OF EFFECTIVE FIELD THEORY}

We were led to the preceding conclusion by assuming the validity of a local effective field theory on the nice time slices. While this assumption is central to the black hole information paradox, it is quite subtle. The nice time slices are locally well behaved but from a global viewpoint they involve very large numbers. The integrated extrinsic curvature along each slice is enormous as can be seen, for example, by parallel transporting a time-like unit normal vector from the region of the outgoing Hawking radiation into the black hole along the radial direction. The resulting vector is related by an enormous local Lorentz boost to a timelike unit normal on this part of the slice. For a large enough black hole we can apply the Rindler space approximation to the near horizon region,

$$
r-r_{s} \ll r_{s},
$$

and simplify the discussion by neglecting the local spacetime curvature. A nice time slice passing through the Rindler region contains observers with large relative boosts from the point of view of the Minkowski time of the region. A minimal condition for the validity of effective field theory is obtained by requiring that a quantum wavepacket, with energy close to some fixed proper cutoff scale $\Lambda$, say $1 \mathrm{TeV}$, and localized near some arbitrary point on the time-slice, not produce a large gravitational back-reaction on the semi-classical geometry, as seen by another wavepacket at some well-separated point [12, 13].

In the Rindler space approximation, one can expect back-reaction to be large when the conditions of the hoop conjecture are satisfied [20]. Namely that horizons form when and only when a mass $M$ can be surrounded by a hoop of circumference $4 \pi G M$ in any direction. Because one can neglect spacetime curvature in the Rindler space limit, notions of circumference and mass are well-defined. 
One criticism that has often been raised against claims of large effects due to large relative boost is that the wavepackets in question are receding from each other rapidly. When these wavepackets are traced backward in time, they converge and at first sight it seems there would have been a large interaction effect. One has similar issues in cosmology, such as the trans-Planckian problem [21]. Usually one takes the viewpoint that modes necessarily started out in the adiabatic vacuum state, where no large effects are present. At much later times, as the cosmological background expands, high frequency modes red-shift down to accessible scales. In this case, populating these modes at late-times need not produce any large effect. In this situation, one can find time-slicings (for example, global time in de Sitter space) where the conditions of the hoop conjecture are only satisfied for wavepackets subject to the usual Jeans instability on sub-horizon size scales, but not for localized modes separated by super-horizon size distances. In the black hole case, the difference is that although the two wavepackets are rapidly receding, nice slices that avoid the singularity allow for wavepackets at small enough separations for the hoop conjecture to apply.

Therefore demanding that the influence of a particle at late-time on the outside not dramatically change the black hole geometry, leads to an upper bound on the allowed relative boost between observers on a given time slice,

$$
\Lambda \gamma_{\max } \ll M
$$

where $M$ is the black hole mass [37]. Our interpretation of this type of the back-reaction bound differs somewhat from that of [12, 13]. We employ the back-reaction bound strictly as a condition for the validity of an effective semiclassical description, allowing for the existence of (small) nonlocal effects even when the bound is satisfied.

The bound (7) is certainly violated on the nice time slices discussed above, where a significant fraction of the Hawking radiation has been emitted, and therefore any conclusions drawn from local effective field theory on such slices should be questioned. In fact, the nonlocal condition on time slices implied by the boost bound restricts the applicability of local effective field theory rather severely in the black hole context. If a time slice intersects both $\mathcal{O}_{\text {free }}$ and $\mathcal{O}_{\text {accel }}$, and we take $\mathcal{O}_{\text {free }}$ to be at Kruskal time $V=1$, then the Kruskal time of $\mathcal{O}_{\text {accel }}$ must satisfy

$$
V=e^{v / r_{s}} \lesssim \gamma_{\max }
$$

in order to respect the boost bound on the time slice in question. The maximum accessible 


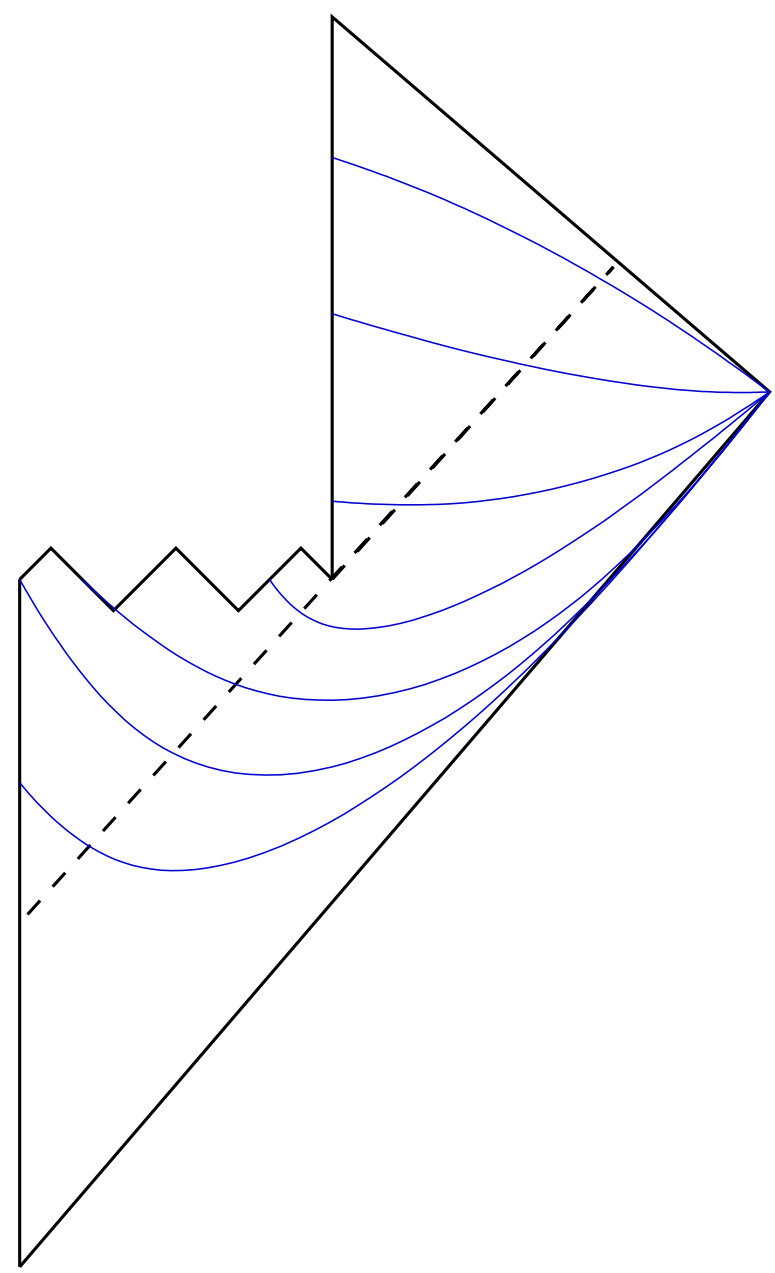

Figure 2: A set of timeslices satisfying the back-reaction bound, and for which an observer hovering close to the horizon remains at low energy.

Schwarzschild time measured by $\mathcal{O}_{\text {accel }}$ before the breakdown of local effective field theory is therefore

$$
t_{\max } \sim r_{s} \log \frac{M}{\Lambda}
$$

This is also roughly the time when the string theory calculation of 11] begins to depart from the corresponding field theory calculation. Other arguments [13, 22, 23] also lead to this time scale.

Since the time (92) is much smaller than the black hole lifetime, $\tau \sim r_{s} S_{b h}$, then according to Page's arguments the information contained in the Hawking radiation at this point is only of order

$$
I \sim e^{-S_{b h}},
$$


where $S_{b h}$ is the initial Bekenstein-Hawking entropy of the black hole.

The Hawking effect was originally derived using local field theory in a black hole background [24], so one might worry that the breakdown of local effective field theory interferes with the Hawking emission process itself. The timescale for the breakdown, given in equation (9), is in fact also the time when the first Hawking photons are emitted from the black hole. However, by our estimate in equation (10), the nonlocal contribution to the amplitude of any given Hawking photon is of order $e^{-S_{b h}}$ so the effect on the average Hawking flux will be negligible.

We note that information retrieval is non-perturbative in $1 / N$ in the AdS/CFT context. Let us apply our estimate to a black hole in $A d S_{5} \times S_{5}$. The relationship between the AdS length scale and parameters of the gauge theory is given by

$$
R^{4}=4 \pi g_{Y M}^{2} N
$$

and for a semiclassical geometry we take $g_{Y M} \rightarrow 0$ and $N \rightarrow \infty$ in such a way that $R \gg 1$ is fixed. A physical length on the gravity side, such as the Schwarzschild radius of a black hole, scales the same way as $R$ with $N$, that is

$$
r_{s} \sim N^{1 / 4}
$$

We are considering black holes with $r_{s} \ll R$, which are localized in the ten-dimensional geometry. The black hole entropy (A3) for $D=10$ scales as

$$
S_{b h} \sim r_{s}^{8} \sim N^{2}
$$

and it follows that $e^{-S_{b h}}$ amounts to a non-perturbative effect in $1 / N$.

The information content (10) is a measure of how much observations of the Hawking radiation perturb the quantum state inside the black hole. From the point of view of the fundamental theory, these really are the same degrees of freedom, so we will use (10) to estimate a lower bound on the size of non-local interactions in the effective field theory. We expect these non-local interactions can be expressed in a form local in time (c.f. the light-cone string field theory calculations of [10, 25, 26]) since the underlying fundamental description, such as the conformal field theory in the case of the AdS/CFT correspondence, is local in time. Note that the asymptotic Schwarzschild time can be used to label the slices. Hence the effective field theory will be nonlocal in space, but otherwise will behave like a conventional quantum mechanical theory. 
The magnitude of the nonlocal effects is of order $e^{-S_{b h}}$, which is far below the intrinsic accuracy of $\mathcal{O}_{\text {free }}$ 's measurements (3), and therefore unobservable. A timelike observer in free fall will hit the singularity within a proper time of order $r_{s}$ after passing through the horizon. The life expectancy of the observer is in fact maximized by following a geodesic in free fall. Any effort at accelerating away from the singularity only serves to reduce the available proper time. The bound we have just established holds along $\mathcal{O}_{\text {free }}$ 's worldline all the way to the singularity. Any time slice that satisfies the boost bound, and on which $\mathcal{O}_{\text {free }}$ is at low energy, can only intersect a small fraction of the outgoing Hawking radiation. Thus only after $\mathcal{O}_{\text {free }}$ has hit the singularity, can $\mathcal{O}_{\text {accel }}$ retrieve substantial information. In this way the maximum boost bound explains why $\mathcal{O}_{\text {free }}$ has a reprieve from burn up until at the singularity, and is free to observe local quantum mechanical evolution to within the accuracy of accessible measurements.

The outside observer $\mathcal{O}_{\text {accel }}$ can detect the tiny amounts of information emitted in the Hawking radiation, since they have access to arbitrarily large measuring apparatus, and in principle, can conduct an arbitrarily large number of measurements on identically prepared black hole states. Thus the non-local effects do act as a one-way transmitter of acausal information, from the inside to the outside of the black hole.

Over the lifetime of the black hole, these small effects are sufficient to emit all the information contained on the inside [16]. One might then worry that at late times, observation of this large amount of information will bring us back to the burn-up scenario for the inside observer. However if $\mathcal{O}_{\text {free }}$ is present on a slice at late-times, we can infer they must have only recently fallen across the horizon. In this case, we can apply Page's formula (10) to bound the information about $\mathcal{O}_{\text {free }}$ emitted in the Hawking radiation, with $S_{b h}$ replaced by the entropy remaining inside the horizon. This allows us to carry over the above estimates of the influence of measurement on the inside observer, to conclude such effects are undetectable on the inside.

This success comes at a price, however. The boost bound restricts the use of effective field theory to foliations of spacetime, for which the time slices terminate at the central singularity from a relatively early time onwards, as indicated in figure 2. To make the effective field theory well-defined it will be necessary to terminate each slice at relatively small spacetime curvature and introduce boundary degrees of freedom to describe the high curvature region. Presumably such a description can be obtained from the AdS/CFT correspondence, but we 
do not know how to carry this out at present. Therefore we content ourselves with placing bounds on the nonlocal effects present in this effective field theory. Alternatively, we could try to formulate an effective theory on a set of nice time slices that avoid the strong coupling region but, since those slices run afoul of the boost bound, unitarity would be implemented in such a theory by nonlocal effects unsuppressed in magnitude.

\section{DISCUSSION}

In quantum gravity, as defined by the AdS/CFT correspondence, black hole evaporation is a unitary process generated by a conventional Hamiltonian on the gauge theory side. The question then becomes how this unitarity is implemented in an effective field theory in a black hole background. The gauge theory correspondence provides a quantum mechanical description of black hole evaporation which is local in time, as measured by distant observers, but the evolution is not local in the bulk spacetime. This is not surprising given that relation between CFT operators and gravitational degrees of freedom is inherently nonlocal, but that relation is of limited direct use due to the weak-to-strong coupling nature of the duality.

Above we estimated the minimal size of nonlocal effects in the effective field theory for information to be returned in the Hawking radiation and made some progress towards characterizing the nonlocality. Interestingly, our order of magnitude estimate for nonlocal effects is comparable to amplitudes involving topology change, computed using the Euclidean path integral formulation of quantum gravity [27, 28].

For an effective field theory picture to remain valid, the family of allowed time slices must satisfy a back-reaction bound. Observation of the Hawking radiation does disturb the state of observers inside the black hole but, due to the bound, the inside observer does not notice information extraction until near the singularity.

The arguments we have given in the present paper offer a more detailed justification of the scenario described in [29], where it was advocated based on the AdS/CFT correspondence that information is extracted from inside observers only as they hit the singularity but not at the horizon. A related idea was put forward in [30] where it was suggested that a unique semiclassical boundary condition should be imposed at the singularity to enforce the extraction of information. It is unclear to us, however, how to impose physical boundary conditions on future spacelike surfaces. In our view the same end is achieved by nonlocal 
dynamical effects as the singularity is approached.

\section{Acknowledgments}

This research is supported in part by DOE grant DE-FG02-91ER40688-Task A and by grants from the Science and Technology Policy Council of Iceland and the University of Iceland Research Fund.

\section{Appendix A: BLACK HOLE GEOMETRY}

The metric of a Schwarzschild black hole in D-dimensional spacetime is given by

$$
d s^{2}=-\left(1-\left(\frac{r_{s}}{r}\right)^{D-3}\right) d t^{2}+\left(1-\left(\frac{r_{s}}{r}\right)^{D-3}\right)^{-1} d r^{2}+r^{2} d \Omega_{D-2}^{2}
$$

where the Schwarzschild radius $r_{s}$ is determined by the product of the D-dimensional Newton's constant and the black hole mass,

$$
r_{s}^{D-3}=\frac{8 \Gamma\left(\frac{D-1}{2}\right)}{(D-2) \pi^{\frac{(D-3)}{2}}} G_{N}^{(D)} M
$$

Some values of $D$ are more interesting than others from the AdS/CFT point of view but it can be useful to have expressions involving arbitrary $D$. Strictly speaking we should be working with a more complicated metric, which reflects the asymptotic AdS nature of the full geometry, but it turns out the Schwarzschild metric (A1) is sufficient for our purposes. We are considering black holes with $r_{s} \ll R$, where $R$ is the AdS length scale, and in this case (A1) provides a good enough approximation in the region of the black hole spacetime that is of interest to us.

The entropy of the black hole is given by $1 / 4$ of the area of the event horizon,

$$
S=\frac{\pi^{\frac{(D-1)}{2}}}{2 \Gamma\left(\frac{(D-1)}{2}\right)} r_{s}^{D-2}
$$

The Hawking temperature of the black hole is most easily obtained by considering the Euclidean continuation $t \rightarrow-i \tau$ of the metric (A1) and requiring the horizon to be smooth,

$$
T_{H}=\frac{(D-3)}{4 \pi r_{s}}
$$


The lifetime $\tau$ of the black hole can be estimated from the Stefan-Boltzmann law,

$$
\frac{d M}{d t} \sim-A T_{H}^{D} \sim-M^{-\frac{2}{D-3}}
$$

from which it follows that

$$
\tau \sim M^{\frac{D-1}{D-3}} \sim r_{s}^{D-1}
$$

The Schwarzschild coordinate system breaks down at the event horizon $r=r_{s}$. For our purposes it is more convenient to work with the D-dimensional analog of Kruskal-Szekeres coordinates. We first introduce a tortoise coordinate $r_{*}$ via

$$
\frac{d r_{*}}{d r}=\left(1-\left(\frac{r_{s}}{r}\right)^{D-3}\right)^{-1}
$$

For $D=4$ this integrates to $r_{*}=r+2 M \log \left[\frac{r}{2 M}-1\right]$ while for $D>4$ we obtain

$$
r_{*}=r+\frac{r_{s}}{D-3} \log \left[\frac{r}{r_{s}}-1\right]+F(r)
$$

where $F(r)$ is finite as $r \rightarrow r_{s}$. The detailed form of $F(r)$ can be figured out but will not be needed here. The next step is to define null coordinates, $v=t+r_{*}$ and $u=t-r_{*}$, and finally these coordinates are exponentiated to obtain

$$
V=\exp \left[\frac{(D-3)}{2 r_{s}} v\right], \quad U=-\exp \left[-\frac{(D-3)}{2 r_{s}} u\right] .
$$

The metric is non-singular at the event horizon in the $(U, V)$ coordinates,

$$
d s^{2}=-\frac{4 r_{s}^{2}}{(D-3)^{2}}\left(\frac{r_{s}}{r}+\left(\frac{r_{s}}{r}\right)^{2} \ldots+\left(\frac{r_{s}}{r}\right)^{D-3}\right) \exp \left[-\frac{(D-3)}{r_{s}}(r+F(r))\right] d U d V
$$

We now have all the ingredients to make an estimate of the timescales involved in the gedanken experiment described in section [II. The curvature singularity at $r=0$ is located on a curve of constant $U V$ in the Kruskal coordinate system. Observer $\mathcal{O}_{\text {accel }}$ waits outside for half the black hole lifetime $t_{1 / 2} \sim r_{s}^{D-1}$ and enters the black hole at retarded Kruskal time $V=V_{0} \sim \exp \left(a^{\prime} r_{s}^{D-2}\right) \sim \exp \left(a S_{b h}\right)$, where $a^{\prime}$ and $a$ are constants of $O(1)$. At this value of $V$ the singularity is at $U=U_{0} \sim 1 / V_{0} \sim \exp \left(-a S_{b h}\right)$, and the worldline of $\mathcal{O}_{\text {accel }}$ will inevitably run into the singularity at an even smaller value of $U$, as can be seen in figure 3.

Observer $\mathcal{O}_{\text {free }}$ is to perform the direct measurement of spin \#1 after entering the black hole and communicate the result to $\mathcal{O}_{\text {accel }}$ by a null signal. This task must be completed 


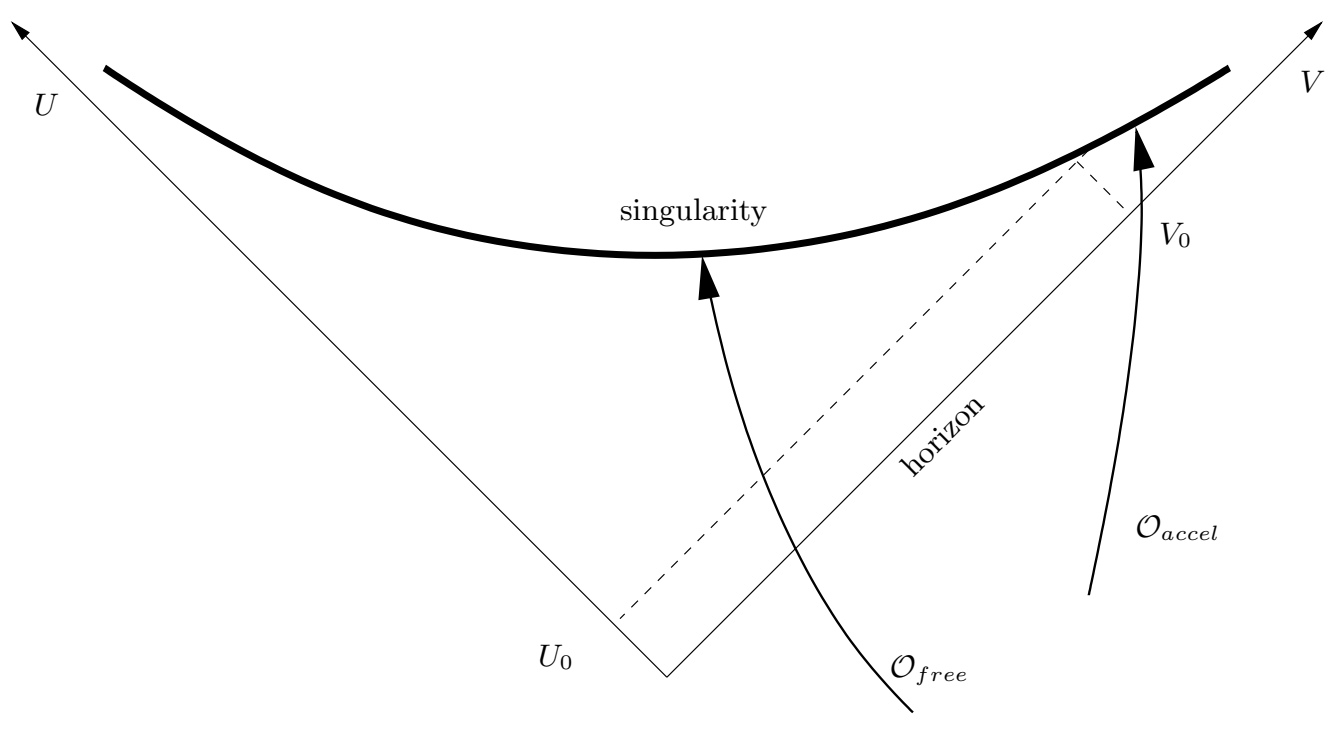

Figure 3: Signal propagation from $\mathcal{O}_{\text {free }}$ to $\mathcal{O}_{\text {accel }}$ is restricted by the black hole geometry.

while $\mathcal{O}_{\text {free }}$ is still in the causal past of $\mathcal{O}_{\text {accel }}$ (see figure 3). It must in other words be carried out on the section of $\mathcal{O}_{\text {free }}$ 's worldline that lies within the narrow range $0<U<U_{0}$. For a large black hole this part of the worldline is in the Rindler region, where spacetime is flat to a good approximation, and the available proper time for $\mathcal{O}_{\text {free }}$ to make the measurement and transmit the result is easily seen to be of order $U_{0} \sim \exp \left(-a S_{b h}\right)$.

It should be noted that there exist black brane geometries in asymptotically AdS spacetime where the timescales involved in estimates of this type appear to allow $\mathcal{O}_{\text {accel }}$ to receive messages from $\mathcal{O}_{\text {free }}$ well before $\mathcal{O}_{\text {accel }}$ runs afoul of the singularity. In all such geometries that we know of, however, the entropy is lower than that of black holes of the same mass density. Such a brane is therefore subject to a Gregory-Laflamme type instability [31, 32] to decay into a collection of black holes on a relatively short timescale and our estimates should instead be applied to the resulting black holes.

\section{Appendix B: AVERAGE ENTROPY OF A SUBSYSTEM}

Consider a quantum system with a Hilbert space of dimension $m n$, which is in a random pure state. Then the average entropy of a subsystem of dimension $m<n$ is given by

$$
S_{m, n}=\sum_{k=n+1}^{m n} \frac{1}{k}-\frac{m-1}{2 n} .
$$


This result was conjectured by Page [15] and later proved by Sen [33]. The information content of the subsystem can be characterized by the difference between $S_{m, n}$ and the maximum thermal entropy

$$
I_{m, n}=\log m-S_{m, n}
$$

The quantum system that we are interested in consists of an uncharged, non-rotating black hole, along with all the Hawking radiation that it has emitted at a given time, as measured by a distant observer in the asymptotic rest frame of the black hole. We take as our subsystem the train of Hawking radiation propagating away from the black hole at a time when the remaining horizon area is more than half that of the original black hole. In this case $n=e^{S_{b h}}$, with $S_{b h}$ the remaining black hole entropy, and we have

$$
n \geq m \gg 1
$$

In this limit the formula (B1) becomes 15

$$
S_{m, n}=\log m-\frac{m}{2 n}+\mathcal{O}\left(\frac{1}{n^{2}}\right)
$$

and the information content of the outgoing radiation is

$$
I_{m, n}=\frac{m}{2 n}+\mathcal{O}\left(\frac{1}{n^{2}}\right)
$$

The subsystem with $m<n$ carries less than one half a bit of information. As described in [16] once the radiation has carried off half the initial entropy of the black hole, the information increases linearly with $\log m$, the number of Hawking photons.

We are also interested in the information content of the Hawking radiation at the onset of evaporation of the black hole. Consider for example the case where the subsystem consists of the polarization degree of freedom of a single Hawking photon only. For $m=2, n \gg m$ (B1) reduces to

$$
S_{2, n}=\log 2-\frac{3}{2 n}+\mathcal{O}\left(\frac{1}{n^{2}}\right)
$$

The average information carried in the polarization of an early Hawking photon is therefore exponentially suppressed,

$$
I_{2, n} \simeq \frac{3}{2 n} \sim e^{-S_{b h}}
$$


where $S_{b h}$ is the initial black hole entropy.

[1] S. W. Hawking, "Breakdown of predictability in gravitational collapse," Phys. Rev. D14 (1976) 2460-2473.

[2] G. 't Hooft, "The black hole horizon as a quantum surface," Phys. Scripta T36 (1991) $247-252$.

[3] L. Susskind, L. Thorlacius, and J. Uglum, "The stretched horizon and black hole complementarity," Phys. Rev. D48 (1993) 3743-3761, hep-th/9306069

[4] T. Banks, W. Fischler, S. H. Shenker, and L. Susskind, "M theory as a matrix model: A conjecture," Phys. Rev. D55 (1997) 5112-5128, hep-th/9610043.

[5] J. M. Maldacena, "The large n limit of superconformal field theories and supergravity," Adv. Theor. Math. Phys. 2 (1998) 231-252, hep-th/9711200.

[6] S. S. Gubser, I. R. Klebanov, and A. M. Polyakov, "Gauge theory correlators from non-critical string theory," Phys. Lett. B428 (1998) 105-114, hep-th/9802109.

[7] E. Witten, "Anti-de sitter space and holography," Adv. Theor. Math. Phys. 2 (1998) 253-291, hep-th/9802150

[8] A. Strominger and C. Vafa, "Microscopic origin of the bekenstein-hawking entropy," Phys. Lett. B379 (1996) 99-104, hep-th/9601029.

[9] R. Wald unpublished (1993).

[10] D. A. Lowe, J. Polchinski, L. Susskind, L. Thorlacius, and J. Uglum, "Black hole complementarity versus locality," Phys. Rev. D52 (1995) 6997-7010, hep-th/9506138

[11] D. A. Lowe, "The planckian conspiracy: String theory and the black hole information paradox," Nucl. Phys. B456 (1995) 257-270, hep-th/9505074

[12] S. B. Giddings and M. Lippert, "Precursors, black holes, and a locality bound," Phys. Rev. D65 (2002) 024006, hep-th/0103231.

[13] S. B. Giddings and M. Lippert, "The information paradox and the locality bound," Phys. Rev. D69 (2004) 124019, hep-th/0402073

[14] L. Susskind and L. Thorlacius, "Gedanken experiments involving black holes," Phys. Rev. D49 (1994) 966-974, hep-th/9308100.

[15] D. N. Page, "Expected entropy of a subsystem," Phys. Rev. Lett. 71 (1993) 1291-1294, 
gr-qc/9305007

[16] D. N. Page, "Information in black hole radiation," Phys. Rev. Lett. 71 (1993) 3743-3746, hep-th/9306083.

[17] W. H. Zurek, "Decoherence and the transition from quantum to classical," Phys. Today 44 (1991) 36-44.

[18] M. Tegmark, "The interpretation of quantum mechanics: Many worlds or many words?," Fortsch. Phys. 46 (1998) 855-862, quant-ph/9709032.

[19] R. Bousso, "A covariant entropy conjecture," JHEP 07 (1999) 004, hep-th/9905177

[20] K. S. Thorne, Magic without Magic: John Archibald Wheeler. San Francisco: Freeman, 1972.

[21] J. Martin and R. H. Brandenberger, "The trans-planckian problem of inflationary cosmology," Phys. Rev. D63 (2001) 123501, hep-th/0005209

[22] K. Schoutens, H. L. Verlinde, and E. P. Verlinde, "Quantum black hole evaporation," Phys. Rev. D48 (1993) 2670-2685, hep-th/9304128.

[23] L. Susskind, "Strings, black holes and lorentz contraction," Phys. Rev. D49 (1994) 6606-6611, hep-th/9308139.

[24] S. W. Hawking, "Particle creation by black holes," Commun. Math. Phys. 43 (1975) $199-220$.

[25] D. A. Lowe, "Causal properties of string field theory," Phys. Lett. B326 (1994) 223-230, hep-th/9312107.

[26] D. A. Lowe, L. Susskind, and J. Uglum, "Information spreading in interacting string field theory," Phys. Lett. B327 (1994) 226-233, hep-th/9402136

[27] J. M. Maldacena, "Eternal black holes in anti-de-sitter," JHEP 04 (2003) 021, hep-th/0106112

[28] S. W. Hawking, "Information loss in black holes," hep-th/0507171.

[29] D. A. Lowe and L. Thorlacius, "Ads/cft and the information paradox," Phys. Rev. D60 (1999) 104012, hep-th/9903237.

[30] G. T. Horowitz and J. Maldacena, "The black hole final state," JHEP 02 (2004) 008, hep-th/0310281.

[31] R. Gregory and R. Laflamme, "Black strings and p-branes are unstable," Phys. Rev. Lett. 70 (1993) 2837-2840, hep-th/9301052.

[32] H. S. Reall, "Classical and thermodynamic stability of black branes," Phys. Rev. D64 (2001) 
044005, hep-th/0104071

[33] S. Sen, "Average entropy of a subsystem," Phys. Rev. Lett. 77 (1996) 1-3, hep-th/9601132.

[34] A. Guijosa and D. A. Lowe, "A new twist on ds/cft," Phys. Rev. D69 (2004) 106008, hep-th/0312282.

[35] D. A. Lowe, "q-deformed de sitter / conformal field theory correspondence," Phys. Rev. D70 (2004) 104002, hep-th/0407188

[36] D. A. Lowe, "Statistical entropy of two-dimensional dilaton de sitter space," Phys. Lett. B624 (2005) 275-280, hep-th/0507001

[37] It is interesting to note that versions of dS/CFT using quantum deformations of the conformal group provide concrete models that realize boost bounds, already at the level of the free theory 34, 35, 36]. 